\title{
Simulation: the power of what hurts
}

D. Turton, K. Buchan, M. Hall-Jackson, C. Pelletier

It is a common adage that we 'learn from our mistakes', and one often cited in the context of Simulation Based Education (SBE). ${ }^{1}$ The study by Bearman et al., ${ }^{2}$ analysing narratives written by people describing their 'most powerful learning experience' in SBE, provides evidence that people certainly do learn from making mistakes. At the same time, however, Bearman et al. caution that 'without appropriate simulation design and facilitation, experiencing error may also damage' learners. ${ }^{2}$ This would appear to put SBE practitioners in a potential bind. Bearman et al. ${ }^{2}$ recommend that, as a response, we might focus on 'things going right' in simulation as much as we already do on 'things going wrong'.

While not denying the benefits of 'learning from success' ${ }^{3}$ and theories such as Safety $I I,{ }^{4}$ we suggest that debriefs are less effective at identifying mistakes than one might imagine and, consequently, that the balance in debriefs is already tipped in favour of focusing on 'things going right'. We submit that the tension between the value of learning from mistakes on the one hand and the risk of causing damage on the other should, in large part, be addressed through consciously developing methods to analyse failures safely.

It is a misconception that debriefing in simulation lends itself to identifying mistakes.

There are reasonable and mutually reinforcing reasons why mistakes are not identified in debriefs. First, facilitators are concerned to make learners feel comfortable and safe. As well as cultural and pedagogic motives for this, there is the practical consideration that learners must be actively engaged in SBE for it to be effective. Pointing out mistakes can alienate learners and hence sabotage the sessions. 
Second, learners may blame their mistakes on the simulation itself ${ }^{1}$ thereby undermining the education value of identifying the mistake and shifting the focus of discussion to the nature of simulation rather than the behaviour in question.

Third, there is pressure, whether explicit or implied, for facilitators to achieve positive feedback because SBE is an expensive resource in ever more burdened healthcare systems. Facilitators may fear that pointing out mistakes might have a negative impact on feedback. Fourth, both facilitators and learners tend to collaborate to reduce the chance of causing harm. To this end, they often avoid identifying, or dwelling upon, mistakes in order to limit the chances of damaging the "professional authority and identity' ${ }^{5}$ of learners. Any such damage represents a significant harm because being viewed as competent, and having trust in one's colleagues, is critical to professional performance.

The desire to avoid causing harm is an expression of professional ethics but also reduces the likelihood of mistakes being identified.

For these reasons, debriefs are often framed in terms of being a non-judgemental examination of non-technical skills, ${ }^{6(267)}$ looking at behaviours not individual performances. Indeed, Pelletier and Kneebone ${ }^{6(\mathrm{p} 269)}$ argue that we create an environment in which mistakes 'cannot be made' because any mistake is reclassified as the demonstration of a specific non-technical skills issue and hence not a 'mistake' at all. A related approach adopted by facilitators is to avoid discussion of mistakes and draw on the concept of 'resilience engineering, ${ }^{7}$ which advocates identifying effective behaviours and then examining how they can be emulated. 
Even when mistakes are identified, it is a challenge

to talk about them, let alone learn from them.

Facilitators legitimately fear misjudging a situation and causing harm to the learner, either due to imperfect communication skills on their part or because of something they are unaware of on the part of the learner. Moreover, both facilitators and learners are aware of the potential for embarrassment and thus any discussion can be so awkward as to be misconstrued or counterproductive.

Ziv et al. argue that 'responses of embarrassment and discomfort should, perhaps, be permitted rather than avoided'1 (p197) but we need to consider how to harness negative emotions while still ensuring the safety of learners, given the potency of the emotions involved.

Mistakes made during a simulation are failures at work; and the experience of failure is suffering.

The work psychologist Dejours ${ }^{8}$ argues that it is precisely the suffering caused by failures at work that drives professional development. The power of this suffering may undermine capacity - the damage referred to by Bearman et al. ${ }^{2}-$ but is also what gives the associated experiences such resonance.

In answer to the challenge of how to manage emotional responses to failure, Rudolph et al. ${ }^{9}$ propose that a sufficiently skilled educator can identify errors without causing negative repercussions by referring to deficits in 'mental models' and 'cognitive frames'. In other words, one can critique behaviour rather than people and this will be well received. We submit that this is unlikely in practice because either we experience failure, however identified, as blame and therefore negatively; or, if it is possible to highlight poor behaviour 
Psychodynamic theory, by contrast, suggests that we use simulation to look at failures in the workplace as defined by the learners and then consider how we can use those experiences to build professional capacity. In this way, mistakes can be put in a social context that means they no longer, necessarily, represent a personal failure but rather a systemic feature limiting the capacity for effective work. This may be one approach by which SBE may offer 'an opportunity for participants to come to understand the role of error in healthcare over time, and to transform thinking about failure to thinking about fallibility', as called for by Bearman et al. ${ }^{2}$

This approach has been piloted at a UK simulation centre, where Pelletier et al. developed scenarios explicitly designed to explore failure at work. ${ }^{5}$ Learners were involved in designing scenarios used to explore the issues, with debriefs to review the simulation and discuss possible responses. Rather than focusing on an individual performance, the learners were swapped in and out of ongoing simulations in order to highlight the collective approach and mitigate against the risk of causing harm to any single individual. During the debrief, failures were contextualised as a function of the workplace and not framed as an individual's limitation. This hopefully made it more likely that they might be discussed and addressed in the future because they were not framed as being an individual's limitation.

We may not be learning from errors as effectively as we imagine and, if so, this represents a missed opportunity.

SBE is now well established within medical education and simulation centres can expand their repertoire to tackle more directly the frustrations in the workplace which hamper performance and ultimately curtail patient care. Bearman et al. describe the 'power' of simulation as 'the learning that resonates long after the experience has passed'. ${ }^{2}$ It is often the failures experienced by learners that resonate most and we should not shy away from 
identifying them and maximising their positive value, exploring experiences in a collective and safe manner. Simulation debriefs offer an opportunity for discussion about shared practice that is unparalleled within medical education and we should strive to find new ways to harness their potential.

Much of the power of simulation - its ability to resonate long after the event - lies precisely in what hurts about simulation. We should seize the opportunity in debriefs to ask not only the safe questions - 'what works?'; but also the challenging ones too - 'what hurts?'

\section{References:}

(1) Ziv A, Ben-David S, Ziv M. Simulation Based Medical Education: an opportunity to learn from errors. Medical Teacher. 2005; 27:3, 193-199.

(2) Bearman M, Greenhill J, Nestel D. The power of simulation: a large-scale narrative analysis of learners' experiences. [preprint] 2018. Medical Education.

(3) Dieckmann P, Patterson M, Lahlou S, Mesman J, Nyström P, Krage R. Variation and adaptation: learning from success in patient safety-oriented simulation training. Advances in Simulation. 2017; 2:21.

(4) Hollnagel E. Safety-I and safety-II: the past and future of safety management. Farnham, Surrey, UK England. Burlington: Ashgate Publishing Company; 2014.

(5) Pelletier C, Buchan K, Hall-Jackson, M. Learning from failure: exploring the psychodynamics of work in a clinical simulation centre. [preprint] 2018. Psychoanalysis, Culture and Society. 
(6) Pelletier C, Kneebone R. Learning safely from error? Reconsidering the ethics of simulation-based medical education through ethnography. Ethnography and Education. 2016; $11: 3,267-282$.

(7) Hollnagel E, Braithwaite J, Wears RL, editors. Resilient Health Care. Farnham: Ashgate; 2013.

(8) Dejours C. Psychopathology of Work: Clinical Observations. London: Karnac Books; 2015.

(9) Rudolph JW, Simon R, Rivard P, Dufresne R, Raemer D. De-briefing with good judgment: combining rigorous feedback with genuine inquiry. Anesthesiology clinics 2007; $25: 2,362-376$. 